Full Length Article

\title{
Factors influencing recruitment and retention of professional nurses, doctors and allied health professionals in rural hospitals in KwaZulu Natal
}

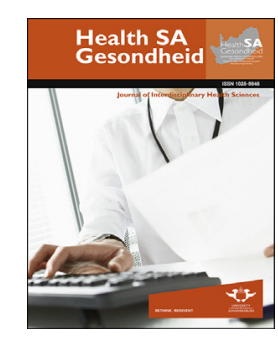

\author{
J. Lyn Haskins*, Sifiso A. Phakathi, Merridy Grant, Christiane M. Horwood \\ Centre for Rural Health, University of KwaZulu-Natal, South Africa
}

\section{A R T I C L E I N F O}

Article history:

Received 20 November 2015

Accepted 7 November 2016

Keywords:

Recruitment

Retention

Professional nurses

Doctors

Allied health professionals

\begin{abstract}
A B S T R A C T
Introduction: In South Africa fewer health professionals (HPs) work in rural areas compared to urban areas, despite rural communities having greater health needs. This study explores factors influencing recruitment and retention of three categories of HPs in KwaZulu-Natal and has implications about how to retain them in rural areas.

Methods: A cross-sectional, descriptive survey was conducted in 8 hospitals, 5 rural and 3 urban, in one district in KZN in 2011. Data were collected on single day in each hospital and all HPs on duty were requested to participate. We compared responses from rural and urban based HP as well as professional nurses (PNs), doctors, and allied HPs.

Results: 417 questionnaires were completed: 150 from HPs in rural and 267 from HPs in urban hospitals. Perceptions of living/working in rural areas is negative and the quality of health care provided in rural areas is perceived as poor by all categories of HP. Rural-based HPs were more likely to report living apart from spouse/partner $(72.1 \%$ vs $37.0 \%, \mathrm{p}<0.001)$ and children $(76.7 \%$ vs $36.9 \%, \mathrm{p}<0.001)$, and living in hospital accommodation $(50.8 \%$ vs $28.9 \% ; \mathrm{p}<0.001)$.

Conclusions: Decisions made by HP about where to work are complex, multifactorial and should be tailored to each category of health professional.
\end{abstract}

(C) 2016 The Authors. Publishing services by Elsevier B.V. on behalf of Johannesburg University. This is an open access article under the CC BY-NC-ND license (http:// creativecommons.org/licenses/by-nc-nd/4.0/).

\section{Introduction and background}

Access to health care is limited by the global shortage of health professionals, particularly in low income countries
(WHO, 2009). Sub-Saharan Africa has the lowest density of health professionals per capita of any region (Kruk et al., 2010), despite having the highest burden of disease (Anyangwe \& Mtonga, 2007; Van Rensburg, 2014). At the same time, the demand for health care is rising as many countries deal with

\footnotetext{
* Corresponding author. Centre for Rural Health, University of KwaZulu-Natal, George Campbell Building, Howard College Campus, Durban 4013, South Africa.

E-mail addresses: haskins@ukzn.ac.za (J.L. Haskins), phakathis@ukzn.ac.za (S.A. Phakathi), grantm1@ukzn.ac.za (M. Grant), christiane@telkomsa.net (C.M. Horwood).

Peer review under responsibility of Johannesburg University. 
an increasing prevalence of both infectious diseases, particularly HIV/AIDS, and chronic diseases of lifestyle (Arnand \& Barnighausen, 2004; Chopra et al., 2009). There is a correlation between the number of health professionals in a particular area, coverage of health services and health outcomes: health professionals improve health and save lives (Anyangwe \& Mtonga, 2007; Arnand \& Barnighausen, 2004). Improvements in health outcomes are dependent on improving access to essential health care. These challenges cannot be met without adequate numbers of health professionals with appropriate skills to provide these interventions.

South Africa is facing epidemic proportions of infectious diseases such as HIV and TB (Van Rensburg, 2014) as well as a rise in non-communicable diseases (Mayosi et al., 2009). This has accelerated the human resource crisis, because of both increasing health care needs and the direct impact on the health workforce of many health workers contracting HIV (Shisana, Hall, Maluleke, Chauveau, \& Schwabe, 2004; Van Rensburg, 2014). Communities in rural and remote areas are the most socioeconomically deprived and have the greatest health needs (Anyangwe \& Mtonga, 2007). However, the particular difficulties in recruiting and retaining skilled health professionals in these areas, has led to disproportionate distribution of health professionals between urban and rural areas. An estimated $46 \%$ of South Africans live in rural areas but only $19 \%$ of all health professionals, and $12 \%$ of doctors, work in these areas (SA Department of South African Department of Health, 2011). As a result, there are critical disparities in access and quality of health care between urban and rural populations, with rural communities being excluded from health benefits available to those in urban areas. If health outcomes are to be improved, it is essential that rural communities have access to skilled health workers in their local areas.

Several descriptive studies have been undertaken to investigate attraction, recruitment and retention of doctors and nurses in rural areas showing there is no single factor responsible, but rather a number of interlinking and complex issues that impact on and influence where HP work (Munyewende, Rispel, \& Chirwa, 2014; Ojakaa, Olango, \& Jarvis, 2014; Steinmetz, de Vries, \& Tijdens, 2014). According to Dolea, Stormont, and Braichet (2010) these factors can be categorized into financial, personal/professional support, education and regulatory factors. A qualitative study in South Africa by Kotzee and Couper (2006) found that increasing the financial remuneration would attract doctors to work in rural areas, while Blaauw et al. (2010) showed that the financial increase would need to be substantial to attract and retain nurses in rural areas. Blaauw et al. (2010) advocate that a locally adapted intervention package to include substantial increases in salary, and improved housing, training, promotion etc., would work to recruit and retain nurses. We found only few studies that influence recruitment and retention among allied health professionals (Hatcher, Onah, Kornik, Peacock, \& Reid, 2014; Morrel, Kiem, Milstead, \& Pollice, 2014).

It is clear, that the development of effective strategies for improving retention of health professionals in rural areas first requires a thorough understanding of the factors that influence health professionals to choose to work in rural areas, and to continue working in these areas.

\section{The aim of the study}

The aim of this study is to investigate factors that influence recruitment and retention of professional nurses, doctors and allied health professionals in rural hospitals in one rural district in KwaZulu-Natal.

\section{Research design and methods}

\subsection{Research design}

A cross sectional study was conducted in all 8 state-funded hospitals in the one district of KwaZulu-Natal. Of the 8 hospitals, 3 were situated in an area classified as urban and 5 in an area classified as rural (see Fig. 1). Hospitals were defined as either urban or rural using the Statistics South Africa definition for rural and urban areas (Statistics South Africa, 2003). Hospitals within municipal boundaries were designated as urban and those outside municipal boundaries were designated as rural.

\subsection{Data collection tool}

A single self-administered, structured questionnaire was developed. The tool was assessed for content validity by passing it through an expert health professional from a rural hospital and pilot tested at a hospital outside of the study site to ensure completeness and usability. Clear instructions to participants were provided on each questionnaire.

\subsection{Data collection methods}

Data were collected on a single $24 \mathrm{~h}$ day. Both night and day staff in each of the participating hospitals and all eligible health professionals on duty at that time, were requested to Eligible health professionals were professional (registered) nurses (PNs), doctors, and allied health professionals. Other nursing cadres (enrolled nurses and enrolled nursing assistants) and lay health workers were excluded.

Data collectors arrived at the hospital, while the night staff were still on duty, and obtained signed informed consent before distributing questionnaires in each ward. HPs completed the questionnaires privately and sealed them in envelopes. Completed questionnaires were later collected by data collectors. The process was repeated for the day staff ensuring that all staff on duty during the $24 \mathrm{~h}$ period had an opportunity to participate.

\subsection{Data analysis}

Data were double entered using EpiData 3.1 and analysis was conducted using Stata 13 for Windows. The Mann Whitney U median value comparison test was used to assess differences in continuous variables between rural and urban based HPs as well as differences between categories of HPs. For categorical 


\begin{tabular}{|c|c|c|c|}
\hline Rural/Urban & Hospital & Number of beds & Hospital designation* \\
\hline \multirow[t]{3}{*}{ Urban Hospitals } & Hospital A & 460 & District hospital \\
\hline & Hospital B & 318 & Specialist referral hospital \\
\hline & Hospital C & 554 & Regional hospital \\
\hline \multirow[t]{5}{*}{ Rural Hospitals } & Hospital D & 170 & District hospital \\
\hline & Hospital E & 212 & District hospital \\
\hline & Hospital F & 141 & District hospital \\
\hline & Hospital G & 120 & District hospital \\
\hline & Hospital H & 162 & District hospital \\
\hline \multicolumn{4}{|c|}{$\begin{array}{l}\text { District hospital definition: A hospital which receives referrals from and provides generalist support to } \\
\text { clinics and community health centres with health treatment administered by general health care } \\
\text { practitioners or primary health care nurses. }\end{array}$} \\
\hline \multicolumn{4}{|c|}{$\begin{array}{l}\text { Regional hospital definition: A hospital which receives referrals from and provides specialist support to } \\
\text { a district hospital and where health care users require the expertise of teams led by resident } \\
\text { specialists. }\end{array}$} \\
\hline
\end{tabular}

Fig. 1 - Description of hospital size and geographical area it serves.

variables the chi-square test was used to determine differences between rural and urban-based HPs as well as differences between categories of HP.

As job satisfaction is closely linked to retention, a job satisfaction survey, adapted from the "Wellness Council of America" survey (Bellingham, 2013), and consisting of 30 questions was included in the questionnaire. A job satisfaction score was calculated from the responses out of a maximum possible score of 60 , and was used to categorise the levels of job satisfaction among respondents. The level of job satisfaction was categorised from the scores as follows: 1-19 very poor; 20-29 poor; 30-39 reasonable; 40-49 good; and $50-60$ very good.

\subsection{Ethical considerations}

Ethical approval for the study was obtained from the University of KwaZulu-Natal (HSS/0209/010) and KwaZulu-Natal Department of Health (HRHM075/10). All participants provided written informed consent after having the study explained to them. Response was voluntary and respondents were not asked to identify themselves on the returned questionnaire ensuring anonymity. Returned questionnaires were kept in a locked room and only aggregate data is reported.

\section{Results}

At the time of data collection, 1011 eligible health professionals were employed in the 8 hospitals, and 417 questionnaires were completed by health professionals on duty. Respondents were grouped into professional nurses (292), medical doctors (46) and allied health professionals (79). Allied health professionals included 21 pharmacists, 17 radiographers, 16 social workers, 8 dieticians, 7 physiotherapists,
5 dentists, 3 occupational therapists, 1 speech therapist and 1 paramedic.

The median age of professional nurses was 43 years (IQR: 34-51) which was significantly older than doctors at 34.5years (IQR: 26-44), $\mathrm{p}<0.001$ and allied health professionals at 29years (IQR: 24-35) p < 0.001. Urban based professional nurses had a median age of 45 years (IQR: 36-53), significantly older than the rural based professional nurses in the study 41years (IQR: 33-49) $p=0.007$. There was no significant difference in the ages of rural and urban based doctors, median age 34 vs $35(p=0.344)$ or between rural and urban based allied health professionals, median age 25 vs 30 ( $p=0.133)$.

The period of time spent working in their respective hospitals was similar for rural and urban-based health professionals ( 6 years vs 5 years: $p=0.608$ ). Professional nurses had worked in the hospital for a median of 9 years (IQR: 4-17), significantly longer than doctors, median 1 year (IQR: 0-3, $\mathrm{p}<0.001)$ and significantly longer than allied health professionals, median 1 year (IQR: $0-5)(p<0.001)$.

Demographic characteristics of participants are shown in Table 1.

\subsection{Social factors}

Professional nurses were equally likely to report having a partner or spouse compared to doctors $(60.5 \%$ vs $76.1 \%$ $\mathrm{p}=0.139)$ and allied health professionals $(48.1 \% \mathrm{p}=0.081)$ but were more likely than doctors $(89.4 \%$ vs $56.5 \% \mathrm{p}<0.001)$ and allied health professionals $(89.4 \%$ vs $54.4 \% \mathrm{p}<0.001)$ to have children Compared to health professionals working in urban areas, those working in rural areas were significantly more likely to report that, while working, they were living apart from their spouse/partner $(72.1 \%$ vs $37.0 \%, \mathrm{p}<0.001)$ and their children (62.4\% vs 22.6, p < 0.001). Rural-based health professionals were also more likely than urban-based health professionals to report that the situation with their spouse/ 
Table 1 - Demographic characteristics of participating health professionals.

\begin{tabular}{|c|c|c|c|c|c|c|c|}
\hline Characteristics & $\begin{array}{c}\text { All health } \\
\text { professionals }\end{array}$ & $\begin{array}{l}\text { Rural } \\
\text { doctors }\end{array}$ & $\begin{array}{l}\text { Rural } \\
\text { allied health } \\
\text { professionals }\end{array}$ & $\begin{array}{c}\text { Rural } \\
\text { professional } \\
\text { nurses }\end{array}$ & $\begin{array}{l}\text { Urban } \\
\text { doctors }\end{array}$ & $\begin{array}{l}\text { Urban } \\
\text { allied health } \\
\text { professionals }\end{array}$ & $\begin{array}{c}\text { Urban } \\
\text { professional } \\
\text { nurses }\end{array}$ \\
\hline Variable & $\mathrm{N}(\%)$ & $\mathrm{n}(\%)$ & $\mathrm{n}(\%)$ & $\mathrm{n}(\%)$ & $\mathrm{n}(\%)$ & $\mathrm{n}(\%)$ & $\mathrm{n}(\%)$ \\
\hline \multicolumn{8}{|l|}{ Age group $(\mathrm{N}=393)$} \\
\hline Younger than 30 years & $88(22.4)$ & 0 & $15(60.0)$ & $15(15.5)$ & $17(43.6)$ & $25(49.0)$ & $16(9.9)$ \\
\hline 30 to 40 years & $119(30.3)$ & $3(60.0)$ & $8(32.0)$ & $37(33.3)$ & $12(30.8)$ & $18(35.3)$ & $41(25.3)$ \\
\hline 41 to 50 years & $103(26.2)$ & $2(40.0)$ & $2(8.0)$ & $38(34.2)$ & $4(10.3)$ & $6(11.8)$ & $53(32.7)$ \\
\hline 51 years and older & $83(21.1)$ & 0 & 0 & 21 (18.9) & $6(15.4)$ & $2(3.9)$ & $52(32.1)$ \\
\hline \multicolumn{8}{|c|}{ Population group $(\mathrm{N}=407)$} \\
\hline African & $358(88.0)$ & $3(60.0)$ & $19(76.0)$ & $119(100)$ & $21(51.2)$ & $35(64.8)$ & $161(97.6)$ \\
\hline White & $27(6.6)$ & 0 & $2(8.0)$ & 0 & $1(2.4)$ & $10(18.5)$ & $2(1.2)$ \\
\hline Indian & $16(3.9)$ & 0 & $4(16.0)$ & 0 & $5(12.2)$ & $6(11.1)$ & $1(0.6)$ \\
\hline Asian & $2(0.5)$ & $2(40.0)$ & 0 & 0 & $13(31.7)$ & 0 & 0 \\
\hline Coloured & $4(1.0)$ & 0 & 0 & 0 & 0 & $2(3.7)$ & $1(0.6)$ \\
\hline \multicolumn{8}{|l|}{ Education $(\mathrm{N}=415)$} \\
\hline Basic qualification & $237(57.1)$ & $4(80.0)$ & $20(83.3)$ & $66(55.5)$ & $24(58.5)$ & $43(79.6)$ & $80(46.5)$ \\
\hline Post basic qualification & $178(42.9)$ & $1(20.0)$ & 4 (16.7) & $53(44.5)$ & $17(41.5)$ & $11(20.4)$ & $92(53.5)$ \\
\hline \multicolumn{8}{|l|}{ Gender $(\mathrm{N}=414)$} \\
\hline Female & 339 (81.9) & 0 & $17(68.0)$ & $103(88.0)$ & $19(46.3)$ & $47(87.0)$ & $153(89.0)$ \\
\hline Male & $75(18.1)$ & $5(100)$ & $8(32.0)$ & $14(12.0)$ & $22(53.7)$ & $7(13.0)$ & $19(11.0)$ \\
\hline \multicolumn{8}{|c|}{ Time working at current hospital $(\mathrm{N}=394)$} \\
\hline$<3$ years & $121(30.7)$ & $4(80.0)$ & $18(72.0)$ & $20(17.5)$ & $27(65.9)$ & $26(48.2)$ & $42(26.9)$ \\
\hline 3 years -6 years & $86(21.8)$ & $1(20.0)$ & $5(20.0)$ & $19(16.7)$ & $10(24.4)$ & $16(29.6)$ & $29(18.6)$ \\
\hline$>6$ years & $187(47.5)$ & 0 & $2(8.0)$ & $75(62.5)$ & $4(9.8)$ & $12(22.2)$ & $85(54.5)$ \\
\hline
\end{tabular}

partner or with their children would encourage them to leave their current workplace (Table 2).

Rural-based health professionals were significantly less likely than those in urban areas to be living in their own home (main residence) while working and more likely to be living in accommodation provided by the hospital (Table 2). Among health professionals living in hospital accommodation, 59.1\% expressed dissatisfaction with their accommodation. Reasons given included lack of privacy, inadequate space, having to share ablutions and bedrooms, and poor maintenance of the

Table 2 - Social and work characteristics reported by participating health professionals.

\begin{tabular}{|c|c|c|c|c|}
\hline Yes responses & Professional nurses & Allied health professionals & Medical doctors & Total \\
\hline \multicolumn{5}{|c|}{ Do you have a spouse or partner? } \\
\hline Total & $\mathrm{n}=292$ & $\mathrm{n}=79$ & $\mathrm{n}=46$ & $\mathrm{~N}=417$ \\
\hline Rural hospitals \% [95\%CI] & $59.7[54.9-64.2]$ & $44[21.8-68.8]$ & 100 & $58.4[52.4-64.2]$ \\
\hline Urban hospitals \% [95\%CI] & $61.1[45.7-74.5]$ & $50[36.0-64.0]$ & $73.2[46.5-89.6]$ & $60.7[50.5-70.0]$ \\
\hline P value & 0.839 & 0.644 & 0.526 & 0.696 \\
\hline \multicolumn{5}{|c|}{ Does your partner or spouse live with you? ${ }^{\text {a }}$ (only HW with spouse/partner) } \\
\hline Total & $\mathrm{n}=176$ & $\mathrm{n}=38$ & $\mathbf{n}=35$ & $\mathrm{~N}=249^{\mathrm{a}}$ \\
\hline Rural hospitals \% [95\%CI] & $27.1[15.6-42.9]$ & $18.2[5.4-46.4]$ & $60.0[14.3-93.1]$ & $27.9[18.2-40.3]$ \\
\hline Urban hospitals \% [95\%CI] & $60.0[51.8-67.7]$ & $63.0[53.7-71.4]$ & $73.3[60.7-83.0]$ & $63.0[57.5-68.1]$ \\
\hline $\mathrm{P}$ value & 0.003 & 0.007 & 0.495 & $<0.001$ \\
\hline \multicolumn{5}{|c|}{ Does the situation with your spouse or partner encourage you to leave the hospital? ${ }^{\text {a }}$ (only HW with spouse/partner) } \\
\hline Total & $\mathrm{n}=176$ & $\mathrm{n}=38$ & $\mathrm{n}=35$ & $\mathrm{~N}=249$ \\
\hline Rural hospitals \% [95\%CI] & $54.2[40.1-67.6]$ & $63.6[38.1-83.3]$ & $60.0[25.8-86.6]$ & $55.9[44.6-66.2]$ \\
\hline Urban hospitals \% [95\%CI] & $19.1[12.8-27.4]$ & $22.2[6.9-52.5]$ & $50.0[26.6-73.4]$ & $25.3[17.5-35.1]$ \\
\hline$P$ value & 0.007 & 0.033 & 0.559 & 0.002 \\
\hline \multicolumn{5}{|l|}{ Do you have any children ${ }^{\mathrm{a}}$} \\
\hline Total & $\mathrm{n}=292$ & $\mathrm{n}=79$ & $\mathrm{n}=46$ & $\mathrm{~N}=417$ \\
\hline Rural hospitals \% [95\%CI] & $84.9[74.9-91.4]$ & $40.0[16.4-69.5]$ & $80.8[57.2-92.3]$ & $77.2[65.3-85.9]$ \\
\hline Urban hospitals \% [95\%CI] & $92.4[86.0-96.1]$ & $61.1[42.9-76.7]$ & $53.7[34.7-71.7]$ & $80.2[65.5-89.6]$ \\
\hline$P$ value & 0.085 & 0.195 & 0.054 & 0.741 \\
\hline \multicolumn{5}{|c|}{ Do your children live with you when you are working? ${ }^{\text {a }}$ (only HW with children) } \\
\hline Total & $\mathrm{n}=260$ & $\mathrm{n}=43$ & $\mathrm{n}=26$ & $\mathrm{~N}=329$ \\
\hline Rural hospitals \% [95\%CI] & $76.2[59.4-87.6]$ & $80.0[34.3-96.8]$ & 100 & $77.4[63.4-87.1]$ \\
\hline Urban hospitals \% [95\%CI] & $35.5[19.8-55.1]$ & $45.5[35.5-56.0]$ & $40.9[17.4-69.5]$ & $37.6[26.0-50.9]$ \\
\hline$P$ value & 0.006 & 0.098 & 0.272 & $<0.001$ \\
\hline
\end{tabular}


Table 2 - (continued)

\begin{tabular}{|c|c|c|c|c|}
\hline Yes responses & Professional nurses & Allied health professionals & Medical doctors & Total \\
\hline \multicolumn{5}{|c|}{ Does the situation with your children encourage you to leave the hospital? ${ }^{\text {a }}$ (only HW with children) } \\
\hline Total & $\mathrm{n}=260$ & $\mathrm{n}=43$ & $\mathrm{n}=26$ & $\mathrm{~N}=329$ \\
\hline Rural hospitals \% [95\%CI] & $58.8[49.8-67.3]$ & $70.0[27.2-93.6]$ & $75.0[41.0-92.8]$ & $60.3[52.1-68.1]$ \\
\hline Urban hospitals \% [95\%CI] & $18.2[8.6-34.6]$ & $36.4[27.2-46.6]$ & $27.3[12.5-49.7]$ & $22.0[13.9-32.9]$ \\
\hline P value & 0.001 & 0.170 & 0.234 & $<0.001$ \\
\hline \multicolumn{5}{|c|}{ Do you consider the place where you live when working to be your main residence?a } \\
\hline Total & $\mathrm{n}=292$ & $\mathrm{n}=79$ & $\mathrm{n}=46$ & $\mathrm{~N}=417$ \\
\hline Rural hospitals \% [95\%CI] & $34.2[25.5-44.0]$ & $4.0[0.4-28.4]$ & $60.0[25.8-86.6]$ & $29.9[22.1-39.1]$ \\
\hline Urban hospitals \% [95\%CI] & $70.2[61.0-78.0]$ & $49.1[33.2-65.1]$ & $57.5[50.1-64.6]$ & $64.0[54.8-72.3]$ \\
\hline P value & $<0.001$ & 0.004 & 0.857 & $<0.001$ \\
\hline \multicolumn{5}{|c|}{ Is your accommodation provided by the hospital? ${ }^{a}$} \\
\hline Total & $\mathrm{n}=292$ & $\mathrm{n}=79$ & $\mathrm{n}=46$ & $\mathrm{~N}=417$ \\
\hline Rural hospitals \% [95\%CI] & $53.0[36.3-69.1]$ & $84.0[60.5-94.7]$ & $80.0[4.7-99.7]$ & $59.2[43.4-73.3]$ \\
\hline Urban hospitals \% [95\%CI] & $12.0[6.0-22.6]$ & $35.9[27.6-45.1]$ & $46.3[26.9-67.0]$ & $22.2[13.5-34.4]$ \\
\hline P value & 0.001 & 0.002 & 0.363 & 0.001 \\
\hline \multicolumn{5}{|c|}{ Are you satisfied with the accommodation supplied by the hospital? ${ }^{a}$ (only HW residing in hospital accommodation) } \\
\hline Total & $\mathrm{n}=82$ & $n=40$ & $\mathrm{n}=23$ & $\mathrm{~N}=145$ \\
\hline Rural hospitals \% [95\%CI] & $32.3[11.2-64.3]$ & $52.4[18.2-84.5]$ & $50.0[50.0-50.0]$ & 37.8 [20.5-58.9] \\
\hline Urban hospitals \% [95\%CI] & $28.0[13.4-49.5]$ & $30.0[6.7-71.8]$ & $52.6[11.5-90.5]$ & $35.9[19.8-56.1]$ \\
\hline$P$ value & 0.288 & 0.378 & 0.886 & 0.693 \\
\hline \multicolumn{5}{|c|}{ Does the situation with accommodation encourage you to leave the hospital? ${ }^{a}$} \\
\hline Total & $\mathrm{n}=292$ & $\mathrm{n}=79$ & $\mathrm{n}=46$ & $\mathrm{~N}=417$ \\
\hline Rural hospitals \% [95\%CI] & $46.7[29.0-65.3]$ & $48.0[15.4-82.4]$ & $40.0[6.9-85.7]$ & $46.7[32.5-61.4]$ \\
\hline Urban hospitals \% [95\%CI] & $29.7[14.9-50.3]$ & $40.7[29.2-53.4]$ & $29.3[5.3-75.5]$ & $31.8[20.8-45.3]$ \\
\hline P value & 0.189 & 0.713 & 0.280 & 0.171 \\
\hline \multicolumn{5}{|c|}{ Are you currently studying towards an additional qualification? ${ }^{a}$} \\
\hline Total & $\mathrm{n}=292$ & $\mathrm{n}=79$ & $\mathrm{n}=46$ & $\mathrm{~N}=417$ \\
\hline Rural hospitals \% [95\%CI] & $12.6[6.5-23.1]$ & $4.0[0.4-28.4]$ & 0.0 & $10.7[6.2-17.9]$ \\
\hline Urban hospitals \% [95\%CI] & $15.8[12.5-19.8]$ & $9.3[0.8-58.8]$ & $20.0[7.2-44.7]$ & $15.2[10.4-21.6]$ \\
\hline$P$ value & 0.454 & 0.538 & 0.582 & 0.278 \\
\hline \multicolumn{5}{|c|}{ Are you able to study towards an additional qualification while working at this hospital? ${ }^{a}$} \\
\hline Total & $\mathrm{n}=292$ & $\mathrm{n}=79$ & $\mathrm{n}=46$ & $\mathrm{~N}=417$ \\
\hline Rural hospitals \% [95\%CI] & $66.7[56.1-75.8]$ & $33.3[21.7-47.5]$ & $20.0[7.7-42.8]$ & $59.4[49.3-68.9]$ \\
\hline Urban hospitals \% [95\%CI] & $66.2[49.1-80.0]$ & $43.1[35.2-51.4]$ & $50.0[35.2-64.8]$ & $58.9[46.6-70.3]$ \\
\hline$P$ value & 0.958 & 0.188 & 0.031 & 0.952 \\
\hline \multicolumn{5}{|c|}{ Does the situation with study opportunities encourage you to leave the hospital? ${ }^{a}$} \\
\hline Total & $\mathrm{n}=292$ & $\mathrm{n}=79$ & $\mathrm{n}=46$ & $N=417$ \\
\hline Rural hospitals \% [95\%CI] & $35.5[29.4-42.2]$ & $71.4[58.8-81.4]$ & 50.0 & $41.7[32.9-51.0]$ \\
\hline Urban hospitals \% [95\%CI] & $42.6[32.9-52.9]$ & $62.2[47.2-75.2]$ & $48.6[22.1-75.9]$ & $47.4[38.9-56.0]$ \\
\hline P value & 0.203 & 0.270 & 0.889 & 0.401 \\
\hline \multicolumn{5}{|c|}{ Are you able to gain experience while working at this hospital? ${ }^{a}$} \\
\hline Total & $\mathrm{n}=292$ & $\mathrm{n}=79$ & $\mathrm{n}=46$ & $\mathrm{~N}=417$ \\
\hline Rural hospitals \% [95\%CI] & $71.6[64.1-78.0]$ & $41.7[15.5-73.6]$ & 100 & $67.6[58.7-75.4]$ \\
\hline Urban hospitals \% [95\%CI] & $82.5[74.9-88.2]$ & $50.9[25.1-76.3]$ & $87.2[62.9-96.5]$ & $76.7[66.5-84.6]$ \\
\hline$P$ value & 0.034 & 0.631 & 0.657 & 0.198 \\
\hline \multicolumn{5}{|c|}{ Does the situation with gaining experience encourage you to leave the hospital? ${ }^{a}$} \\
\hline Total & $\mathrm{n}=292$ & $\mathrm{n}=79$ & $\mathrm{n}=46$ & $\mathrm{~N}=417$ \\
\hline Rural hospitals \% [95\%CI] & $52.5[41.4-63.3]$ & $32.0[9.8,67.2]$ & $40.0[13.4-74.2]$ & $48.7[38.9-58.6]$ \\
\hline Urban hospitals \% [95\%CI] & $69.2[63.2-74.6]$ & $37.0[19.6-58.6]$ & $68.3[41.0-87.0]$ & $62.6[53.4-70.9]$ \\
\hline P value & 0.008 & 0.771 & 0.010 & 0.033 \\
\hline
\end{tabular}

accommodation. Levels of dissatisfaction with accommodation were similar among urban and rural based health professionals, and professional nurses, doctors and allied health professionals were all equally likely to state that they were unhappy with their accommodation (Table 2).

Table 3 shows the perceptions of HPs living and working in a rural area. Both rural and urban based health professions expressed the opinion that remuneration is better and promotion is easier to obtain in rural hospitals. They also felt patients experienced a better quality of care in urban hospitals.

\subsection{Work experience and study opportunities}

Most health professionals working in both rural and urban areas $(84.2 \%)$ said they would like to obtain an additional 
Table 3 - Perceptions of participants about living and working in a rural area.

\begin{tabular}{|c|c|c|c|c|}
\hline Variables & $\begin{array}{c}\text { Rural-based health } \\
\text { professionals } \mathrm{N}=150\end{array}$ & $\begin{array}{c}\text { Urban-based health } \\
\text { professionals } \mathrm{N}=267\end{array}$ & All health professionals $\mathrm{N}=417$ & $\begin{array}{l}\chi \text { squared } \\
\text { P-value }\end{array}$ \\
\hline $\begin{array}{l}\text { Agreement with the } \\
\text { statement }\end{array}$ & $\mathrm{n}(\%)$ & $\mathrm{n}(\%)$ & $\mathrm{n}(\%)$ & \\
\hline \multicolumn{5}{|c|}{ Social and accommodation factors } \\
\hline $\begin{array}{l}\text { Schooling for children in } \\
\text { rural areas is as good as in } \\
\text { urban areas }\end{array}$ & $11(7.5)$ & $39(15.1)$ & $50(12.4)$ & 0.033 \\
\hline $\begin{array}{l}\text { My family situation will } \\
\text { make me leave my } \\
\text { present employment } \\
\text { within the next } 6 \text { months }\end{array}$ & $50(33.3)$ & $53(19.9)$ & $103(24.7)$ & 0.008 \\
\hline $\begin{array}{l}\text { Living in a urban area costs } \\
\text { more than living in a rural } \\
\text { area }\end{array}$ & $106(70.7)$ & $212(79.4)$ & $318(76.3)$ & 0.045 \\
\hline $\begin{array}{l}\text { Having a group friends in } \\
\text { the area makes me stay in } \\
\text { my present employment }\end{array}$ & $56(37.3)$ & $116(43.4)$ & $172(41.2)$ & 0.321 \\
\hline $\begin{array}{l}\text { Housing in rural areas is } \\
\text { good }\end{array}$ & $35(24.0)$ & 85 (32.9) & $120(29.7)$ & 0.044 \\
\hline $\begin{array}{l}\text { Working in a rural area } \\
\text { would appeal to me }\end{array}$ & $67(54.0)$ & $119(50.4)$ & $186(51.7)$ & 0.566 \\
\hline \multicolumn{5}{|l|}{ Quality of life } \\
\hline $\begin{array}{l}\text { The quality of life in a rural } \\
\text { area is very good }\end{array}$ & $35(23.8)$ & $83(32.0)$ & $118(29.1)$ & 0.169 \\
\hline $\begin{array}{l}\text { Cost of living is lower in } \\
\text { rural areas }\end{array}$ & $89(59.7)$ & $172(66.4)$ & $261(64.0)$ & 0.267 \\
\hline $\begin{array}{l}\text { I would like to work in a } \\
\text { urban hospital }\end{array}$ & $96(64.0)$ & $166(62.2)$ & $262(62.8)$ & 0.739 \\
\hline $\begin{array}{l}\text { I would move to a rural area } \\
\text { for more money }\end{array}$ & $53(35.8)$ & $117(44.8)$ & $170(41.6)$ & 0.270 \\
\hline $\begin{array}{l}\text { It is not safe to travel } \\
\text { around in a rural areas }\end{array}$ & $69(46.6)$ & $121(46.2)$ & $190(46.3)$ & 0.933 \\
\hline $\begin{array}{l}\text { You can earn more money } \\
\text { in rural hospitals }\end{array}$ & $57(39.3)$ & $158(61.2)$ & $215(53.3)$ & 0.015 \\
\hline \multicolumn{5}{|l|}{ Work and work experience } \\
\hline $\begin{array}{l}\text { Work load is heavier in rural } \\
\text { hospitals }\end{array}$ & $96(64.0)$ & $157(58.8)$ & $253(60.7)$ & 0.530 \\
\hline $\begin{array}{l}\text { It is easier to get promotion } \\
\text { in rural hospitals }\end{array}$ & $43(29.5)$ & $143(55.0)$ & $186(45.8)$ & $<0.001$ \\
\hline $\begin{array}{l}\text { There is a shortage of } \\
\text { professional health } \\
\text { workers in rural hospitals }\end{array}$ & $124(82.7)$ & $232(86.9)$ & $356(85.4)$ & 0.141 \\
\hline $\begin{array}{l}\text { Working in rural hospitals } \\
\text { means you lack the } \\
\text { support of colleagues }\end{array}$ & $48(32.9)$ & $83(31.7)$ & $131(32.1)$ & 0.874 \\
\hline $\begin{array}{l}\text { Patients get better quality of } \\
\text { care in urban hospitals }\end{array}$ & $99(66.0)$ & $202(75.7)$ & $301(72.2)$ & 0.027 \\
\hline $\begin{array}{l}\text { Communication in a rural } \\
\text { hospital is poor }\end{array}$ & $89(59.3)$ & $161(60.3)$ & $250(60.0)$ & 0.883 \\
\hline $\begin{array}{l}\text { There is not enough } \\
\text { essential equipment in a } \\
\text { rural hospitals }\end{array}$ & $123(82.0)$ & $221(82.8)$ & $344(82.5)$ & 0.828 \\
\hline $\begin{array}{l}\text { You get more experience } \\
\text { working in an urban } \\
\text { hospital }\end{array}$ & $118(78.7)$ & $215(80.5)$ & $333(79.9)$ & 0.608 \\
\hline \multicolumn{5}{|l|}{ Study and study opportunities } \\
\hline $\begin{array}{l}\text { When working in a rural } \\
\text { hospital there is more } \\
\text { time to study }\end{array}$ & $44(30.1)$ & $106(40.8)$ & $150(36.9)$ & 0.042 \\
\hline
\end{tabular}

qualification, including further diplomas (49.2\%), bachelor degrees (6.7\%), master degrees (22.5\%), doctoral degrees (3.8\%) and other non-medical qualifications (1.2\%). Among health professionals wishing to obtain further qualifications, most reported being able to undertake their studies while working at their current hospital (Table 2).

The majority of the health professionals (73.5\%) said they were able to gain work experience at their current hospital. This 
was highest among doctors (88.6\%), but not significantly different from professional nurses $(75.3 \% \mathrm{p}=0.164)$. There was a significant difference between doctors and allied health professionals (88.6\% vs $48.1 \%, p=0.003$ ) in their ability to gain work experience at their current hospital, similarly between professional nurses and allied health professionals $(75.3 \%$ vs $48.1 \%, p=0.006)$.

\subsection{Job satisfaction}

The mean job satisfaction score for all respondents was 37.5 and was similar for rural and urban health professionals (37.5 vs $37.5, p=0.839$ ). There was a significant difference between the job satisfaction scores of professional nurses and doctors ( 37.6 vs $43.7 \mathrm{p}=0.002$ ) and between doctors and allied health professionals (43.7 vs 33.7, $\mathrm{p}<0.001$ ).

Overall the job satisfaction score showed that $46.8 \%$ of respondents expressed good or very good levels of job satisfaction, $24.9 \%$ scored reasonable and $28.3 \%$ had a job satisfaction score that was bad or very bad. A summary of job satisfaction criterion are shown in Table 4.

\section{Discussion}

Although this is a small study it is unique in that it compares perceptions of HP in rural and urban areas as well as considers attitudes and perceptions between various HP, namely professional nurses, doctors and allied health professionals.

Findings show a number of contradictory views and perceptions among the three categories of HPs. Perceptions of life in rural areas are negative, even among rural-based health professionals, often associated with environmental factors such as schools, housing and safety being perceived as inferior in rural areas which is corroborated by previous findings Dolea et al. (2010) and Ojakaa et al. (2014). In addition, the quality of health care in rural areas was considered as poor by

Table 4 - Responses of participants to job satisfaction survey questions.

\begin{tabular}{|c|c|c|c|c|}
\hline Statement & Rural Hospitals $n=150$ & Urban Hospitals $n=267$ & All $\mathrm{N}=417$ & $\chi$ squared \\
\hline Job attribute & $\mathrm{n}(\%)$ & $\mathrm{n}(\%)$ & $\mathrm{n}(\%)$ & \\
\hline $\begin{array}{l}\text { I look forward to going to work on a Monday } \\
\text { morning }\end{array}$ & $98(68.5)$ & $177(69.4)$ & $275(69.1)$ & 0.922 \\
\hline Work is a real plus in my life & $128(87.7)$ & $205(80.1)$ & $333(82.8)$ & 0.171 \\
\hline I feel valued \& affirmed at work & $89(60.5)$ & $153(60.2)$ & $242(60.3)$ & 0.958 \\
\hline I am engaged in meaningful work & 131 (89.7) & $216(86.1)$ & $347(87.4)$ & 0.402 \\
\hline $\begin{array}{l}\text { I am positive \& up most of the time I am } \\
\text { working }\end{array}$ & $114(79.7)$ & $202(79.2)$ & $316(79.4)$ & 0.929 \\
\hline I am fairly compensated & $65(44.5)$ & $102(40.2)$ & $167(41.8)$ & 0.183 \\
\hline $\begin{array}{l}\text { I have energy at the end of the day to engage } \\
\text { in personal interests }\end{array}$ & $70(47.6)$ & $110(43.1)$ & $180(44.8)$ & 0.582 \\
\hline Most interactions at work are positive & $87(60.4)$ & $168(65.9)$ & $255(63.9)$ & 0.452 \\
\hline My values fit with the organizational values & $91(64.1)$ & $157(63.3)$ & $248(63.6)$ & 0.898 \\
\hline $\begin{array}{l}\text { I have the materials \& equipment that I need } \\
\text { in order to do my work }\end{array}$ & $41(28.5)$ & $111(43.9)$ & $152(38.3)$ & 0.216 \\
\hline My manager cares for me as a person & $89(61.4)$ & $149(60.1)$ & $238(60.6)$ & 0.813 \\
\hline My manager reviews my progress & $98(67.6)$ & $157(62.3)$ & $255(64.2)$ & 0.421 \\
\hline I know what is expected of me at work & $140(95.2)$ & $238(93.0)$ & $378(93.8)$ & 0.431 \\
\hline $\begin{array}{l}\text { I have the opportunity to do what I do best } \\
\text { every day at work }\end{array}$ & $93(65.0)$ & $161(64.1)$ & $254(64.5)$ & 0.367 \\
\hline $\begin{array}{l}\text { I have energy at the end of each working day } \\
\text { for the people I care about }\end{array}$ & $85(58.2)$ & $119(46.7)$ & $204(50.9)$ & 0.173 \\
\hline $\begin{array}{l}\text { I have the time and energy in my life to read } \\
\text { books that interest me }\end{array}$ & $69(47.3)$ & $117(45.5)$ & $186(46.2)$ & 0.763 \\
\hline I have good friends at work & $116(71.5)$ & $217(84.8)$ & $333(82.8)$ & 0.204 \\
\hline I feel recognised and appreciated at work & $77(52.0)$ & $146(57.5)$ & $223(55.5)$ & 0.374 \\
\hline I feel free to be who I am & $115(79.3)$ & $193(75.1)$ & $308(76.6)$ & 0.218 \\
\hline I feel free to do things the way I like at work & $50(34.5)$ & $102(39.7)$ & $152(37.8)$ & 0.575 \\
\hline My co-workers are committed to quality work & $131(91.0)$ & $198(79.5)$ & $329(83.7)$ & 0.026 \\
\hline I am aligned with the organisational mission & $125(87.4)$ & $211(84.7)$ & $336(85.7)$ & 0.476 \\
\hline I trust our leadership team & $90(62.1)$ & $150(60.2)$ & $240(60.9)$ & 0.743 \\
\hline I respect the work of my peers & $141(95.9)$ & $240(95.2)$ & $381(95.5)$ & 0.797 \\
\hline $\begin{array}{l}\text { I have opportunities to learn what I want to } \\
\text { learn }\end{array}$ & $69(48.3)$ & $134(52.5)$ & $203(51.0)$ & 0.551 \\
\hline $\begin{array}{l}\text { I feel involved in decisions that involve our } \\
\text { organisational community }\end{array}$ & $50(34.2)$ & $102(40.2)$ & $152(38.0)$ & 0.167 \\
\hline Creativity and innovation are supported & $70(49.3)$ & $140(56.0)$ & $210(53.6)$ & 0.350 \\
\hline I feel informed of what's going on & $72(50.0)$ & $143(56.7)$ & $215(54.3)$ & 0.392 \\
\hline My opinions count & $76(52.8)$ & $151(59.9)$ & $227(54.4)$ & 0.321 \\
\hline $\begin{array}{l}\text { I know someone at work who encourages my } \\
\text { development }\end{array}$ & $105(71.9)$ & $173(68.4)$ & $278(69.7)$ & 0.642 \\
\hline
\end{tabular}


all categories of health professionals, problems of inadequate equipment, shortage of staff and heavy workloads were reported similar to those described by Van Rensburg (2014). Despite these attitudes, we found high levels of job satisfaction, with good career opportunities which were similar in both urban and rural areas and among all categories of health workers. These positive factors however did not influence and determine job choices among our participants. This supports the findings of other researchers that state that despite the challenges faced working in a rural district hospital, there can still be high levels of job satisfaction (Hegney \& McCarthy, 2000; Munyewende et al., 2014).

In addition, our findings suggest health workers in rural areas were much more likely to be living away from their families (partner/spouse and children) and in hospital accommodation, a situation which frequently led to dissatisfaction and a desire to leave their current post which correlates to findings of Steinmetz et al. (2014) who found having children at home increases turnover of nurses. Paradoxically, professional nurses working in rural hospitals were much older than doctors and allied health professionals, and provided a relatively stable workforce, having worked at their current hospital for a medium duration of more than 10 years also corroborating findings of Steinmetz et al. (2014). It is unclear why professional nurses who express dissatisfaction chose to work for long periods in rural hospitals, despite the many challenges they expressed and further research into this phenomenon should be considered. Nurses make up a large proportion of the workforce and interventions should be put in place to retain them.

Doctors and allied health professionals were younger, less likely to have children to consider, and were a more mobile workforce. The median length of time spent working in their current hospital among this cadres was just over three years, and it was likely that many of them were undertaking their compulsory community service. This suggests strategies for retaining and recruiting doctors and allied health professionals to rural areas should be developed differently from nurses. Planning for hospital accommodation should specifically provide appropriate accommodation for younger, single health professionals as well as for families with children.

This study suggests that decisions made by health professionals regarding whether to work in an urban or a rural area are complex and multi-factorial, and include both work and social factors. The general environment in rural areas, as well as the living and working conditions, combine to make rural areas a challenging place to work. Similar to other studies, remuneration remains a factor in the decision making process, which is influenced by both push and pull factors (Willis-Shattuck, Bidwell, Thomas, Blaauw and Ditlopo, 2008). Improved remuneration and higher grade posts in rural areas may tend to pull health workers to these areas, but will be mitigated by the push factors, like poor working and living conditions, which have a negative effect on retention of these staff as previously described.

A country's ability to recruit and retain health care professionals in underserved areas ultimately depends upon the provision of a stable, rewarding and fulfilling personal and professional environment (Hart, Salsberg, Phillips, \& Lishner, 2002) and a broad range of factors can influence recruitment and retention of health professionals including poor remuneration, poor job satisfaction, lack of development opportunities, and poor living and working conditions (Blaauw et al., 2013). This has resulted in a number of interventions in South Africa aimed at improving staffing levels in rural hospitals and clinics, and to reduce mal-distribution of health care professionals (Ditlopo, Blaauw, Rispel, Thomas, \& Bidwell, 2013; Hatcher, Onah, Kornik, Peacock, \& Reid, 2014). These have included financial incentives, and improved opportunities for training and career advancement (WillisShattuck et al., 2008). Our findings suggest that financial incentives have led to perceptions that remuneration is better and promotion easier to obtain in rural hospitals. A high proportion of health professionals expressed that they would move to a rural area for more money, indicating that this remains an important factor. However, these interventions have not been sufficient to provide adequate staffing in rural areas. The effectiveness of current interventions has not been formally evaluated, and there are few well-designed studies to evaluate any of the numerous interventions that have been implemented to address the shortage of health care professionals practicing in rural areas (Grobler et al., 2009). In the meantime, staffing in rural facilities remains inadequate and governments are urged to implement interventions which cover the wide and complex variety of factors including improved working conditions, living conditions, professional development opportunities and remuneration.

\section{Limitations}

There were a number of limitations to the study. The study was undertaken in only one district of KwaZulu-Natal where urban hospitals that were included were much larger and fundamentally different from the rural hospitals that were included, and therefore, findings cannot be broadly generalised. In addition, the number of respondents for doctors and allied health professionals in rural hospitals were smaller than urban hospitals limiting the ability to draw strong conclusions. Despite these shortcomings, this study alludes to a number of important aspects about working in rural settings and provides local insight to where interventions are needed to address dissatisfaction before health professionals leave their current posts.

\section{Recommendations}

Based on the findings of this study we have a few important recommendations.

- Firstly to attract health professional to work in rural areas accommodation conducive to family living should be provided.

- Health professionals see the quality of health care in rural areas as poor. If this perception is improved it may attract health professions to the rural areas. This could include improvement in the equipment provided in rural hospitals, improved staffing for rural hospitals, which may in turn lead to reduction of heavy workloads 
- It would be important to tailor incentives to the different categories of health workers. For example doctors may want improved accommodation to attract them to rural areas while allied health professionals or professional nurses may prefer higher incomes.

- Further research is needed to evaluate the effectiveness of single interventions to the different categories of health professionals versus bundles of interventions.

\section{Conclusions}

It is likely that the complex interlinking of different factors impact on recruitment and retention and means no single solution will solve the problem. Therefore, a bundle of interlinked interventions may be more effective as advocated by Blaauw and colleagues [11]. Additional support for health professionals in rural areas to live in these areas with their families could be implemented to not only attract health professionals to these areas but also to retain them. Provision of good housing and support for school fees for children, would both provide financial incentives for health professionals as well as mitigating some of the negative factors identified. Providing a good experience of working in rural areas for young doctors and allied health professionals may encourage them to stay and the provision of suitable housing would go a long way to do this. However, further incentives should be piloted and carefully evaluated before being rolled out to ensure that interventions are cost effective and do not have unwanted side effects.

\section{Authors contributions}

LH, SP, MG and CH were all involved in the conceptualisation of the study. LH and SP were involved in data collection. SP and $\mathrm{CH}$ undertook the data analysis. $\mathrm{LH}, \mathrm{SP}$ and $\mathrm{CH}$ drafted the manuscript and all authors approved the final draft of the manuscript.

\section{Acknowledgements}

We would like to thank the research team Mr Lucky Nzimande, Mr Innocent Buthelezi and Ms Zama Mthethwa for assisting in conceptualizing the study and collecting the data. We would also thank the District Manager, CEO's of all 8 hospitals and the individual health professionals for their participation.

\section{R E F E R E N C E S}

Anyangwe, S. C. E., \& Mtonga, C. (2007). Inequities in the global health workforce: The greatest impediment to health in subSaharan Africa. Internation Journal Environmental Research Public Health, 4(2), 93-100. http://www.ncbi.nlm.nih.gov/pmc/ articles/PMC3728573/ (Accessed 9 September 2013).

Arnand, S., \& Barnighausen, T. (2004). Human resources and health outcomes: Cross-country econometric study. The Lancet, 365, 1603-1609 [PMID:15519630].
Bellingham R.. The wellness council of America. Job satisfaction survey. http://www.welcoa.org/freeresources/index.php? category=11 (Accessed 9 September 2013).

Blaauw, D., Ditlopo, P., Maseko, F., Chirwa, M., Mwisongo, A., Bidwell, P., et al. (2013). Comparing the job satisfaction and intention to leave of different categories of health workers in Tanzania, Malawi, and South Africa. Global Health Action, 6 [PMID:23364090].

Blaauw, D., Erasmus, E., Pagaiya, N., Tangcharoensathein, V., Mullei, K., Mudhune, S., et al. (2010). Policy interventions that attract nurses to rural areas: A multicountry discrete choice experiment. Bulletin of the World Health Organisation, 88, 350-356. http://dx.doi.org/10.2471/BLT.09.072918.

Chopra, M., Lawn, J. E., Saunders, D., Barron, P., Abdool Karim, S. S., Bradshaw, D., et al. (September 2009). Achieving the health millennium development goals for South Africa: Challenges and priorities. The Lancet, 373(9694), 1023-1031.

Ditlopo, P., Blaauw, D., Rispel, L. C., Thomas, S., \& Bidwell, P. (2013). Policy implementation and financial incentives for nurses in South Africa: A case study on the occupationspecific dispensation. Global Health Action, 6 [PMID:23364040].

Dolea, C., Stormont, L., \& Braichet, J. M. (2010). Evaluated strategies to increase attraction and retention of health workers in remote and rural areas. Bulletin of the World Health Organisation, 88, 379-385. http://dx.doi.org/10.2471/ BLT.09.070607.

Grobler, L., Marais, B. J., Mabunda, S. A., Marindi, P. N., Reuter, H., \& Volmink, J. (2009 Jan 21). Interventions for increasing the proportion of health professionals practising in rural and other underserved areas. Cochrane Database Systematic Review, 1, CD005314 [PMID:19160251].

Hart, G., Salsberg, E., Phillips, D. M., \& Lishner, D. M. (2002). Rural health care providers in the United States. Journal of Rural Health, 18(5), 211-232 [PMID:12061515].

Hatcher, A. M., Onah, M., Kornik, S., Peacock, J., \& Reid, S. (2014). Placement, support and retention of health professionals: National, cross sectional findings from medical and dental community service officers in South Africa. Human Resources for Health, 12, 14. http://dx.doi.org/10.1186/1478-4491-12-14.

Hegney, D., \& McCarthy, A. (July/August 2000). Job satisfaction and nurses in rural Australia. Journal of Nursing Administration, 30(7/8), 347-350 [PMID:10953692].

Kotzee, T., \& Couper, I. D. (2006). What interventions do South African qualified doctors think will retain them in rural hospitals of the Limpopo province of South Africa. Rural and Remote Health, 6, 581 (Online). Available from: http://rrh. deakin.edu.au (Accessed 13 July 2014).

Kruk, M. E., Johnson, J. C., Gyakobo, M., Agyel-Baffour, P., Asabir, K., Kotha, S. R., et al. (2010). Rural practice preference among medical students in Ghana: A discrete choice experiment. Bulletin of the World Health Organ, 88, 333-341.

Mayosi, B. M., Flisher, A. J., Lalloo, U. G., Sitas, F., Tollman, S. M., \& Bradshaw, D. (2009). The burden of non-communicable diseases in South Africa. The Lancet, 384(9693), 934-937. http:// dx.doi.org/10.1016/S0140-6736(09)61087-4.

Morrel, A. L., Kiem, S., Milstead, M. A., \& Pollice, A. (2014). Attraction, recruitment and distribution of health professionals in rural and remote Australia: Early results of the Rural Health Professionals program. Human Resources for Health, 12, 15. http://dx.doi.org/10.1186/1478-4491-12-15.

Munyewende, P. O., Rispel, L. C., \& Chirwa, T. (2014). Positive practice environments influence job satisfaction of primary health care clinic nursing managers in two South African provinces. Human Resources for Health, 12, 27. http://dx.doi.org/ 10.1186/1478-4491-12-27. Available from: http://www.humanresources-health.com/content/12/1/27.

Ojakaa, D., Olango, S., \& Jarvis, J. (2014). Factors affecting motivation and retention of primary health care workers in 
three disparate regions in Kenya. Human Resources for Health, 12, 33. http://dx.doi.org/10.1186/1478-4491-12-33.

Shisana, O., Hall, E. J., Maluleke, R., Chauveau, J., \& Schwabe, C. (2004). HIV/AIDS prevalence among South African health workers. South African Medical Journal, 94(10), 846-850 [PMID: 15532762].

South African Department of Health. (2011). Human resources for health South Africa: HRH strategy for the health sector 2012/ 13-2016/17. SA DOH http://www.doh.gov.za/docs/reports/ 2013/Human_resources.pdf (Accessed 5 September 2013). Statistics South Africa. (2003). Census 2001: Investigation into appropriate definitions of urban and rural areas for South Africa: Discussion document. Pretoria: Statistics South Africa. http:// www.statssa.gov.za/census01/html/UrbanRural.pdf (Accessed 5 September 2013).

Steinmetz, S., de Vries, D. H., \& Tijdens, K. G. (2014). Should I stay or should I go? The impact of working time and wages on retention in the health workforce. Human Resources for Health,
12, 23. http://dx.doi.org/10.1186/1478-4491-12-23. Available from: http://www.human-resources-health.com/content/12/ 1/23 (Accessed 1 June 2016).

Van Rensburg, H. C. J. (2014). South Africa's protracted struggle for equal distribution and equitable access - still not there. Human Resources for Health, 12, 26. http://dx.doi.org/10.1186/ 1478-4491-12-26. Available from: http://www.humanresources-health.com/content/12/1/26 (Accessed 1 June 2016).

WHO. (2009). Increasing access to health workers in remote and rural areas through improved retention. Global policy recommendations. World Health Organization http:// whqlibdoc.who.int/publications/2010/9789241564014_eng.pdf (Accessed 5 September 2013).

Willis-Shattuck, M., Bidwell, P., Thomas, S., Wyness, L., Blaauw, D., \& Ditlopo, P. (2008). Motivation and retention of health workers in developing countries: A systematic review. BMC Health Services Research, 8, 247 [PMID:19055827]. 